\title{
AN ADJOINT REPRESENTATION FOR POLYNOMIAL ALGEBRAS
}

\author{
STEPHEN A. MITCHELL AND ROBERT E. STONG
}

\begin{abstract}
This paper shows that a graded polynomial algebra over $F_{2}$ with Steenrod algebra action possesses an analog of the adjoint representation for the cohomology of the classifying space of a compact connected Lie group.
\end{abstract}

1. Introduction. Let $R=F_{2}\left[z_{1}, z_{2}, \ldots, z_{n}\right]$ be a graded polynomial algebra over the field with two elements and, in addition, suppose that $R$ has an action of the mod 2 Steenrod algebra $A$ for which it is an unstable algebra over $A$. This condition means that $R$ has the formal algebraic properties satisfied by the mod 2 cohomology of a space. Specifically, there are homomorphisms $\mathrm{Sq}^{i}: R^{j} \rightarrow R^{j+i}$ for $i \geq 0$ satisfying

$$
\text { (1) (Cartan formula) } \mathrm{Sq}^{i}(x y)=\sum_{j=0}^{i} \mathrm{Sq}^{j} x \cdot \mathrm{Sq}^{i-j} y
$$

and

$$
\text { (2) (Unstability) } \mathrm{Sq}^{i} x= \begin{cases}x & \text { if } i=0, \\ x^{2} & \text { if } i=\operatorname{dim} x, \\ 0 & \text { if } i>\operatorname{dim} x .\end{cases}
$$

It is convenient to let $\mathrm{Sq}=1+\mathrm{Sq}^{1}+\mathrm{Sq}^{2}+\cdots$ be the total Steenrod square, which is then an algebra automorphism of $R$. As examples we have:

(1) Let $R_{0}=F_{2}\left[x_{1}, \ldots, x_{n}\right]$ with $\operatorname{dim} x_{i}=1$. The action of $A$ is then uniquely described by $\operatorname{Sq} x_{i}=x_{i}+x_{i}^{2}$, and $R_{0}$ is isomorphic to $H^{*}\left(B Z_{2}^{n} ; Z_{2}\right)$, the $\bmod 2$ cohomology of the classifying space for the group $Z_{2}^{n}$.

(2) Let $R_{1}=\left(F_{2}\left[x_{1}, \ldots, x_{n}\right]\right)^{\Sigma_{n}}$ be the ring of invariants of the symmetric group acting to permute the $x_{i}$. Then $R_{1}=F_{2}\left[w_{1}, \ldots, w_{n}\right]$, where $w_{i}$ is the $i$ th elementary symmetric function of the $x_{j}$. Then $R_{1}$ is isomorphic to $H^{*}\left(B O(n) ; Z_{2}\right)$, where $O(n)$ is the orthogonal group, with $w_{i}$ being called the $i$ th universal Stiefel-Whitney class.

(3) Let $R_{2}=D_{n}=\left(F_{2}\left[x_{1}, \ldots, x_{n}\right]\right)^{\mathrm{GL}_{n} F_{2}}$ be the ring of invariants of the general linear group $\mathrm{GL}_{n} F_{2}$, considered as the linear transformations of the span of $x_{1}, \ldots, x_{n}$. Then $D_{n}=F_{2}\left[c_{n^{\prime} n-1}, \ldots, c_{n^{\prime} 0}\right]$, where $\operatorname{dim} c_{n^{\prime} i}=2^{n}-2^{i}$, is called the $n$th Dickson algebra. Except for small values of $n$ it is not the $\bmod 2$ cohomology of a space.

The prototypical example is, of course, given by $R=H^{*}\left(B G ; Z_{2}\right)$, which is the mod 2 cohomology of the classifying space of a compact Lie group. For general $G$ the cohomology is not polynomial, but we are considering the polynomial situation. In this situation, with $R=F_{2}\left[z_{1}, \ldots, z_{n}\right], n$ is the $\bmod 2$ rank of $G$ (see $[\mathbf{Q}]$ ) and

Received by the editors April 1, 1986.

1980 Mathematics Subject Classification (1985 Revision). Primary 55R40. 
$m=\sum_{1}^{n}\left(\operatorname{dim} z_{i}-1\right)$ is the dimension of $G$. In analogy we can call $n$ and $m$ the rank and dimension of $R$.

When $G$ is a compact Lie group, we have the adjoint representation giving a homomorphism Ad: $G \rightarrow O(m)$. This induces a map $B$ Ad: $B G \rightarrow B O(m)$, with an induced homomorphism $H^{*}\left(B O(m) ; Z_{2}\right) \rightarrow H^{*}\left(B G ; Z_{2}\right)$ or equivalently a homomorphism

$$
\text { Ad: } F_{2}\left[w_{1}, w_{2}, \ldots, w_{m}\right] \rightarrow R \text {. }
$$

The objective of this paper is to show that such a homomorphism actually arises directly from the algebraic structure of $R$. In particular, a polynomial algebra which is an unstable algebra over the mod 2 Steenrod algebra has a homomorphism

$$
\text { Ad: } F_{2}\left[w_{1}, \ldots, w_{m}\right] \rightarrow R=F_{2}\left[z_{1}, \ldots, z_{n}\right]
$$

which is an analogue of the adjoint representation.

The authors are indebted to the National Science Foundation for financial support during this work.

2. A definition. If $G$ is a compact Lie group, we have the adjoint representation Ad: $G \rightarrow O(m)$ and hence an induced homomorphism Ad: $H^{*} B O(m) \rightarrow H^{*} B G$, where we let $H^{*} B G=H^{*}\left(B G ; Z_{2}\right)$. Of course, one way to obtain this homomorphism is to consider the representation Ad as giving a vector bundle $E G \times_{G} R^{m} \rightarrow$ $E G / G=B G$. This vector bundle has a Thom space $T \operatorname{Ad}_{G}$, and the reduced mod 2 cohomology of that Thom space $H^{*} T \operatorname{Ad}_{G}$ is a free $H^{*} B G$ module of rank one, with generator a Thom class $U \in H^{m} T \operatorname{Ad}_{G}$. Then $\operatorname{Sq} U=\left(1+w_{1}+\cdots+w_{m}\right) U$, where the class $w_{i}$ is obtained by writing $\operatorname{Sq}^{i} U$ as a multiple of $U$.

More generally, a homomorphism $H^{*} B O(m) \rightarrow R$ always arises from a Thom module over $R$, i.e. a module over the semitensor product $r \odot A$ that is free of rank one as an $R$-module (Handel $[\mathbf{H}]$ ). We are then asking for a Thom module for $R$ analogous to the adjoint Thom module $H^{*} T \operatorname{Ad}_{G}$ for $H^{*} B G$.

THEOREM. Let $R$ be a polynomial algebra of rank $n$ and dimension $m$ over the mod 2 Steenrod algebra. Then there is a Thom module $\operatorname{Ad}_{R}$ over $R$ with the following properties:

(a) If $R=H^{*} B G$, where $G$ is either a compact connected Lie group or $O(n)$, then $\operatorname{Ad}_{R} \cong H^{*} T \operatorname{Ad}_{G}$.

(b) $\operatorname{dim} \operatorname{Ad}_{R} \leq m$; i.e. $w_{i}=0$ if $i>m$,

(c) If $Q$ is another polynomial A-algebra, $\operatorname{Ad}_{Q \otimes R}=\operatorname{Ad}_{Q} \otimes \operatorname{Ad}_{R}$.

(d) $\operatorname{Ad}_{R}$ is trivial (i.e. $w_{i}=0$ for $i>0$ ) if and only if $R$ is abelian.

(e) If $T$ is abelian and $R \rightarrow T$ is a nonsingular embedding with Jacobian determinant $J$, then $\operatorname{Ad}_{R} \cong R \cdot J \subset T$.

REMARKS. The meaning of (d) and (e) will be explained in the course of the proof. The groups other than $O(n)$ to which (a) applies are $S O(n), U(n), S U(n)$, $\operatorname{Sp}(n), \operatorname{Spin}(n)$ for $n \leq 9, G_{2}, F_{4}$, and products of these (Borel [B]). While no details will be given, there are $\bmod p$ analogues when $p$ is odd prime.

To define $\operatorname{Ad}_{R}$ we make use of the Hochschild homology $H_{*} R$, which is defined to be $\operatorname{Tor}^{R \otimes R}(R, R)$ (see [C-E]). By standard homological methods $H_{*} R$ becomes a commutative (in the graded sense) $R \odot A$-algebra whenever $R$ is an $A$-algebra (recall that $\mathrm{Sq}$ is an algebra automorphism). If $R$ is a polynomial of rank $n$, we define $\operatorname{Ad}_{R}=H_{n} R$. Now $\operatorname{Ad}_{R}$ is certainly a module over $R \odot A$, but we must 
explain why it is free of rank one as an $R$-module. In fact, when $R$ is a polynomial, $H_{*} R$ can be described very simply as follows: Let $\Omega_{R}^{1}$ denote the module of 1 forms on $R$-i.e., the free $R$-module on symbols $d x, x \in R$, modulo the relation: $d(x y)=x d y+y d x$. Thus if $z_{1}, \ldots, z_{n}$ are polynomial generators for $R, \Omega_{R}^{1}$ is a free $R$-module on $d z_{1}, \ldots, d z_{n}$. Furthermore, defining $\operatorname{Sq}(d x)=d \operatorname{Sq} x, \Omega_{R}^{1}$ becomes a module over $R \odot A$. Hence the algebra $\Omega_{R}^{*}$ of differential forms becomes an $R \odot A$ algebra. Note: $\Omega_{R}^{k}$ is free of rank $\left(\begin{array}{l}n \\ k\end{array}\right)$ as an $R$-module.

PROPOSITION. $\Omega_{R}^{*} \cong H_{*} R$ as $R \odot A$-algebras.

ProOF. Define $\phi^{1}: \Omega_{R}^{1} \rightarrow H_{1} R$ by mapping $a d x$ to the class of $a \otimes x$ in the Hochschild complex (see Loday-Quillen [L-Q]). It is easy to see that this is an isomorphism of $R$-modules (for any commutative algebra $R$ ) and hence, in our context, an isomorphism of $R \odot A$-modules. Since $H_{*} R$ is strictly anticommutative with respect to the Hochschild grading $\left(x \in H_{k} R, k\right.$ odd, then $\left.x^{2}=0\right), \phi^{1}$ extends to a homomorphism $\phi: \Omega_{R}^{*} \rightarrow H_{*} R$ of $R \odot A$-algebras. On the other hand, by using a Koszul resolution to compute $H_{*} R$, it is easy to see that $\phi$ is an isomorphism. (Indeed, this is a trivial special case of a theorem of Hochschild, Kostant, and Rosenberg [H-K-R].) Thus $H_{n} R=\Omega_{R}^{n}$ is a Thom module with "Thom class" $d z_{1} d z_{2} \cdots d z_{n}$ (see also [B-S]).

PROOF OF THE THEOREM. (a) Let $G_{c}$ denote $G$ regarded as a left $G$-space via the conjugation action: $g \cdot x=g x g^{-1}$. Then it is easy to see that there is a pullback diagram up to homotopy

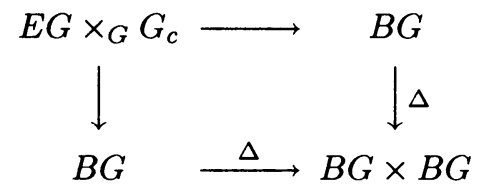

where $\Delta$ is the diagonal map. (Thus, $E G \times{ }_{G} G_{c}$ is homotopy equivalent to the free loop space on $B G$; see [S2].) Now if $G$ is connected, so that $B G \times B G$ is simply connected, the Eilenberg-Moore spectral sequence associated to (1) converges to $H^{*}\left(E G \times_{G} G_{c}\right)$. Its $E_{2}$-term is precisely $H_{*} R\left(R=H^{*} B G\right)$, and it is a spectral sequence of $A$-algebras. Furthermore, the spectral sequence collapses, since it is a second-quadrant cohomology spectral sequence and $H_{*} R$ is generated by $H_{0} R=R$ and $H_{1} R$. Note that $H_{n} R$ is therefore a quotient of $H^{*}\left(E G \times_{G} G_{c}\right)$. Now $\operatorname{Ad}_{G}=$ $E G \times_{G} \operatorname{ad}_{G}$, where $\operatorname{ad}_{G}$ is the adjoint representation of $G$. Identifying $\operatorname{ad}_{G}$ with a $G$-invariant neighborhood $U$ of the identity in $G_{c}$, we obtain a collapse map

$$
E G \times_{G} G_{c} \stackrel{h}{\rightarrow} E G^{+} \wedge_{G}(U / \partial U) \cong T\left(\operatorname{Ad}_{G}\right) .
$$

Let $f$ denote the composite $H^{*} T\left(\operatorname{Ad}_{G}\right) \stackrel{h^{*}}{\longrightarrow} H^{*}\left(E G \times_{G} G_{c}\right) \stackrel{\pi}{\rightarrow} H_{n} R$, where $\pi$ is the projection. Then $f$ is a map of $R \odot A$-modules, and it only remains to show that $f$ maps Thom class to Thom class.

To see this, consider the commutative diagram

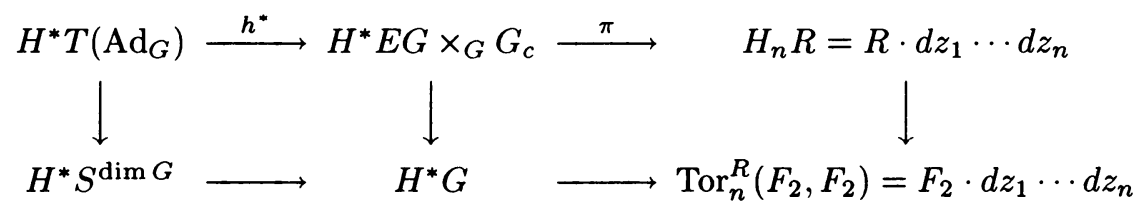


where the left-hand square arises from the diagram

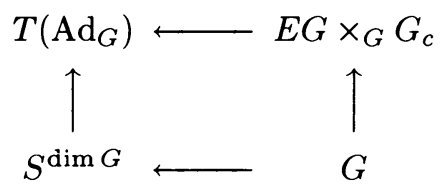

and the right-hand one from the map of Eilenberg-Moore spectral sequences obtained by mapping the pullback diagram

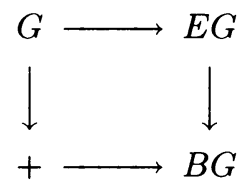

into the diagram (1). Here $S^{k}$ is the sphere of dimension $k$.

The spectral sequence of (3) also collapses. Furthermore, it is easy to see that the corresponding map $H_{*} R \rightarrow \operatorname{Tor}^{R}\left(F_{2}, F_{2}\right)$ coincides with the projection $H_{*} R \rightarrow$ $\left(H_{*} R\right) / \bar{R} H_{*} R$, where $\bar{R}$ is the augmentation ideal. In particular, the map $H_{n} R \rightarrow$ $\operatorname{Tor}_{n}^{R}\left(F_{2} F_{2}\right)$ is surjective. Since the bottom row of $(2)$ is an isomorphism, it follows that $\pi h^{*}$ is an isomorphism, as desired.

The case $G=O(n)$ is easy to check directly - see the example below.

(b) Let $R=F_{2}\left[z_{1}, \ldots, z_{n}\right]$, where $\left|z_{i}\right|=d_{i}$. Then $\operatorname{Sq}^{d_{i}}\left(d z_{i}\right)=d\left(z_{i}^{2}\right)=0$. Hence $\operatorname{Sq}\left(d z_{1} \cdots d z_{n}\right)=0$ if $k>r$.

(c) This is a general property of Hochschild homology.

Now let $U=F_{2}\left[x_{1}, \ldots, x_{n}\right], \operatorname{dim} x_{i}=1$, with its unique unstable $A$-algebra structure. An $A$-algebra $R$ will be called abelian if $R$ is isomorphic to a subalgebra of $U$ of the form $F_{2}\left[x_{1}^{2^{i}} 1, \ldots, x_{n}^{2^{i} n}\right]$ for some nonnegative integers $i_{1}, \ldots, i_{n}$. If $R$ is abelian, then clearly $\operatorname{Ad}_{R}$ is trivial. An embedding of polynomial $A$-algebras

$$
R=F_{2}\left[z_{1}, \ldots, z_{n}\right] \hookrightarrow T=F_{2}\left[y_{1}, \ldots, y_{n}\right]
$$

is nonsingular if the Jacobian determinant $J=\operatorname{det}\left[\partial z_{i} / \partial y_{j}\right]$ is nonzero.

(e) An embedding $R \stackrel{i}{\rightarrow} T$ is nonsingular if and only if the induced map $H_{n} R \stackrel{i_{n}}{\longrightarrow}$ $H_{n} T$ is nonzero, in which case $i_{*}$ is an isomorphism onto $R \cdot\left(J d y_{1} \cdots d y_{n}\right)$. But if $T$ is abelian, this Thom module can be identified with $R \cdot J \subset T$ (in particular $R \cdot J$ is a sub- $A$-module of $T$ ).

Next, one knows from the work of Adams and Wilkerson $[\mathbf{A}-\mathbf{W}]$ that any $R$ of rank $n$ can be embedded in $U$. Of course, the embedding can be singular, but on inspection, it appears that this can only happen for trivial reasons-i.e., $R \subset T$ for some proper abelian subalgebra $T$ of $U$. This led us to conjecture that every $R$ admits a nonsingular embedding in some abelian $T$ (of the same rank, of course); this conjecture has been proven by Wilkerson $[\mathbf{W}]$. This result is of interest in its own right, but here we simply note that part (d) of the theorem follows immediately: Choose a nonsingular embedding of $R$ in an abelian $T$. Then $\mathrm{Ad}_{R} \cong R \cdot J$ as in (e), and if $J \neq 1$ (i.e., $R \neq T$ ) then $A$ acts nontrivially on $J$. This completes the proof of the Theorem.

EXAMPLES. (a) Let $R=H^{*} B O(n)=U^{\Sigma_{n}}=F_{2}\left[w_{1}, \ldots, w_{n}\right]$. Then $J$ is easily seen to be the Vandermonde determinant $\prod_{i<j}\left(x_{i}+x_{j}\right)$. Hence Sq $J=$ $\left(\prod_{i<j} 1+x_{i}+x_{j}\right) \cdot J$ and $w\left(\operatorname{Ad}_{R}\right)=\prod_{i<j}\left(1+x_{i}+x_{j}\right)($ see $[\mathbf{B}-\mathbf{H}, \S 15.4])$. 
(b) Let $R=U^{\mathrm{GL}_{n} F_{2}}$ be the Dickson algebra. Then $R=F_{2}\left[e_{1}, \ldots, e_{n}\right]$, where $e_{i}=c_{n, n-i}$, and the $A$-action is given by

$$
\mathrm{Sq} e_{i}=\left(e_{i}+\cdots+e_{n}\right)\left(1+e_{1}+\cdots+e_{n}\right)+\sum_{j>1} e_{j}^{2} .
$$

Then $w\left(\operatorname{Ad}_{R}\right)=\left(1+e_{1}+\cdots+e_{n}\right)^{n-1}$. This follows either directly, or by computing $J=e_{n}^{n-1}$. For example, if $n=3$ then $R \cong H^{*}\left(B G_{2}\right)$, and we have $w\left(\operatorname{Ad}_{G_{2}}\right)=$ $1+e_{1}^{2}+e_{2}^{2}+e_{3}^{2}$. (The formula for Sq $e_{i}$ can be derived using the methods of $[\mathbf{W}]$.)

REMARK. The most interesting case is when $R \subset U$ is already a nonsingular (i.e., separable) embedding. In that case, we see that $\operatorname{dim} \operatorname{Ad}_{R}$ is precisely $r$.

3. An alternative definition. Let $R=F_{2}\left[z_{1}, \ldots, z_{n}\right]$ be a polynomial algebra which is an unstable algebra over $A$, and let $S=F_{2}\left[u_{1}, \ldots, u_{n}\right]$ be a subalgebra invariant under $A$ and having the same rank. Then $R \otimes_{S} F_{2}=R / R S^{+}$, which we denote $R / S$, is a Poincaré duality algebra over the Steenrod algebra of dimension $N=\operatorname{dim} S-\operatorname{dim} R=\sum \operatorname{dim} u_{i}-\sum \operatorname{dim} z_{i}$. Let $\pi: R \rightarrow R / S$ be the quotient homomorphism. (See $[\mathbf{S 1}]$.)

Since $R / S$ is a Poincaré duality algebra, it has normal Stiefel-Whitney classes $\bar{w}_{i}(R / S) \in(R / S)^{i}$ characterized by $\bar{w}_{i}(R / S) x=\left(\mathrm{Sq}^{-1} x\right)^{n}$ for all $x \in(R / S)^{n-i}$. These are the Stiefel-Whitney classes corresponding to the contragredient module $(R / S)^{\#}$, called the normal Thom module.

PROPOSITION. There are unique classes $\bar{w}_{i} \in R^{i}$ having the property that for every subalgebra $S \subset R$ of the same rank, $\pi \bar{w}_{i}=\bar{w}_{i}(R / S)$ is the normal StiefelWhitney class.

PROOF. $R^{2^{s}}=F_{2}\left[z_{1}^{2^{s}}, \ldots, z_{n}^{2^{s}}\right]$ is a subalgebra for which $\pi: R^{i} \rightarrow\left(R / R^{2^{s}}\right)^{i}$ is an isomorphism if $i<2^{s}$, and taking $2^{s}>i$ determines the class $\bar{w}_{i}$ by its projection. To see that this is well defined, we have inclusions

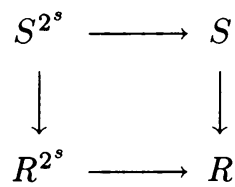

for any subalgebra $S$, with $R^{2^{s}} / S^{2^{s}} \rightarrow R / S^{2^{s}} \stackrel{\pi_{1}}{\longrightarrow} R / R^{2^{s}}$ and $S / S^{2^{s}} \rightarrow R / S^{2^{s}} \stackrel{\pi_{2}}{\longrightarrow}$ $R / S$ being Poincaré extensions in the sense of [M, Appendix B]. Then $\bar{w}_{i}\left(R / S^{2^{s}}\right)$ and the projection of $\bar{w}_{i}$ in $R / S^{2^{s}}$ have the same image under $\pi_{1}$ [M, Proposition B1], so coincide, since $2^{s}>i$; and then applying $\pi_{2}$ yields that $\bar{w}_{i}(R / S)$ is the projection of $\bar{w}_{i}$ into $R / S$, since it coincides with the projection of $\bar{w}_{i}\left(R / S^{2^{s}}\right)$.

Proposition. In $R, \bar{w}_{j}=0$ if $j>\operatorname{dim} R$.

ProOF. According to Adams-Wilkerson $[\mathbf{A}-\mathbf{W}]$, we have an inclusion $R \subset$ $U=F_{2}\left[x_{1}, \ldots, x_{n}\right]$ with $\operatorname{dim} x_{i}=1$, where $U$ is the algebraic closure of $R$. By $[\mathbf{M}$, Theorem B3], $\bar{w}(U)=1$. Using standard notation we let $[M]$ denote the fundamental homology class of the Poincaré algebra $M$. We may then choose a class $a \in U$ so that $\pi(a)[U / R]=1$. Then in the sequence

$$
R / R^{2^{s}} \stackrel{i}{\rightarrow} U / R^{2^{s}} \stackrel{\pi}{\rightarrow} U / R
$$


we have $r\left[R / R^{2^{s}}\right]=\operatorname{air}\left[U / R^{2^{s}}\right]$ and so

$$
\begin{aligned}
\bar{w}(R) r^{\prime}\left[R / R^{2^{s}}\right] & =\mathrm{Sq}^{-1} r^{\prime}\left[R / R^{2^{s}}\right]=a i \mathrm{Sq}^{-1} r^{\prime}\left[U / R^{2^{s}}\right] \\
& =\mathrm{Sq}\left(a i \mathrm{Sq}^{-1} r^{\prime}\right)\left[U / R^{2^{s}}\right]
\end{aligned}
$$

since $\bar{w}(U)=1$, and this is Sq $a \cdot i r^{\prime}\left[U / R^{2^{s}}\right]$. Since the largest nonzero term of Sq $a$ is in dimension $2 \operatorname{dim}(U / R)$, we have $\bar{w}(R) r^{\prime}\left[R / R^{2^{s}}\right]=0$ if $\operatorname{dim} r^{\prime}<\operatorname{dim}\left(R / R^{2^{s}}\right)-$ $\operatorname{dim}(U / R)$, or $\bar{w}_{j}(R)=0$ if $j>\operatorname{dim}(U / R)$.

COROLlaRY. The classes $\bar{w}_{i}$ define a homomorphism $H^{*} B O(m) \rightarrow R$.

PrOOF. We clearly have a homomorphism $F_{2}\left[w_{1}, \ldots, w_{m}\right] \rightarrow R$ with $w_{i}$ mapping to $\bar{w}_{i}$. Projecting into the Poincaré algebra $R / R^{2^{s}}$ for large $s$, the images are Stiefel-Whitney classes of a Poincaré algebra and satisfy all relations true in $H^{*} B O(m)$.

Proposition. If $R=H^{*} B H$ and $S \subset R$ is the subalgebra $H^{*} B G$ for some compact Lie group $G$ containing $H$, then the Stiefel-Whitney class of the adjoint bundle $w\left(\operatorname{Ad}_{H}\right)$ restricts to $\bar{w}(R / S)$ in $R / S$.

Proof. In the fibring $G / H \stackrel{i}{\rightarrow} B H \rightarrow B G$ it is well known that the bundle $\operatorname{Ad}_{H}$ restricts to the normal bundle of the manifold $G / H$. Thus $\pi\left(w\left(\operatorname{Ad}_{H}\right)\right)=$ $i^{*} w\left(\operatorname{Ad}_{H}\right) \in R / S=H^{*} G / H$ is the Stiefel-Whitney class of the normal Thom module of $R / S$.

Of course, when $R=H^{*} B H$ there are not enough subalgebras $S \subset R$ of the form $H^{*} B G$ with $H \subset G$ to make this lead to a proof that $\bar{w}(R)=w\left(\operatorname{Ad}_{H}\right)$. By extensive and unpleasant calculation we have verified this equality for all $H$ known to have $H^{*} B H$ polynomial. It is pointless to reproduce such calculations. Instead we are led to

PROBLEM. Does the class $\bar{w} \in R$ defined by using subalgebras coincide with the class $w\left(\mathrm{Ad}_{R}\right)$ arising from Hochschild homology?

\section{REFERENCES}

[A-W] J. F. Adams and C. W. Wilkerson, Finite $H$-spaces and algebras over the Steenrod algebra, Ann. of Math. (2) 111 (1980), 95-143.

[B] A. Borel, Sous-groupes commutatifs et torsions des groupes de Lie compacts connexes, Tôhoku Math. J. 13 (1961), 216-240.

[B-H] A. Borel and F. Hirzebruch, Characteristic classes and homogeneous spaces. I, Amer. J. Math. 80 (1958), 458-538.

[B-S] P. Baum and L. Smith, The real cohomology of differentiable fiber bındles, Comment. Math. Helv. 42 (1967), 171-179.

[C-E] H. Cartan and S. Eilenberg, Homological algebra, Princeton Univ. Press, Princeton, N.J., 1956.

[H] D. Handel, Thom modules, J. Pure Appl. Algebra 36 (1985), 237-252.

[H-K-R] G. Hochschild, B. Kostant and A. Rosenberg, Differential forms on regular affine algebras, Trans. Amer. Math. Soc. 102 (1962), 383-408.

[L-Q] J. L. Loday and D. G. Quillen, Cyclic homology and the Lie algebra homology of matrices, Comment. Math. Helv. 59 (1984), 565-591.

[M] S. A. Mitchell, Finite complexes with A(n)-free cohomology, Topology 24 (1985), 227-246.

[Q] D. G. Quillen, The spectrum of an equivariant cohomology ring. I, II, Ann. of Math. (2) 94 (1971), 549-602. 
[S1] L. Smith, A note on the realization of graded complete intersection algebras by the cohomology of a space, Quart. J. Math. Oxford. 33 (1982), 379-384.

[S2] - On the characteristic zero cohomology of the free loop space, Amer. J. Math. 103 (1981), 887-910.

[W] C. W. Wilkerson, Inseparable forms of algebras over the Steenrod algebra, preprint.

Department of Mathematics, University of Washington, Seattle, WashingTON 98195

Department of Mathematics, University of Virginia, Charlottesville, VirGINIA 22903 